\title{
The Role of Social Media in the Emotional Lives of People with Vitiligo
}

\author{
A Thesis Presented to: \\ The Faculty of the Department of Sociology \\ University of Oregon and The McNair Scholars Program \\ In Partial Fulfillment of: \\ The Requirements of the Degree of Bachelor of Science
}

Elizabeth Witcher 


\begin{abstract}
Previous research on new media has shown that social media may help to bring people of marginalized groups together for greater support across geographical lines. The current study expanded on previous research by analyzing emotional experiences and compiling themes in relation to the use of Instagram by people with Vitiligo. Vitiligo is a highly visual, often stigmatizing, common skin condition that causes loss of pigmentation (color) which affects up to $2 \%$ of the population globally regardless of race or gender. Instagram was picked for this study due to its visual nature. This application allows the use of hashtags as a search tool for possible connections with others. The researcher interviewed people with Vitiligo who use Instagram about their experiences to better answer the queries related to the role of social media in the lives of people with Vitiligo. It was found that this community works on weak ties. Participants took solace in the presence of others, rather than making strong connections.
\end{abstract}




\section{The Role of Social Media in the Emotional Lives of People with Vitiligo}

"So when I got Instagram, I started to put the pictures up. It was like being welcomed into a community. It was like so many people. It was like people were waiting for me to show up, you know what I mean? It felt really good." -Christian

\section{Introduction}

Vitiligo has been becoming more mainstream in the media recently. There is a new public figure Winnie Harlow (Chantelle Brown-Young) with Vitiligo who is working as a model and activist to spread awareness. She was involved in various things but her career really took off in 2014 when she was cast on America's Next Top Model for Cycle 21. Show host and creator Tyra Banks found Winnie through Instagram where she already had a huge presence. Winnie did not make it as far as to win America's Next Top Model, but she did manage to keep the momentum going. She became an international model helping bring Vitiligo into the mainstream. During the writing of this thesis she secured campaigns for well known labels such as Converse, Coach, Marc Jacobs, and Nike. Ads could be seen of her with prominent Vitiligo across various big cities. Gap ran a campaign for a collaboration with Disney for their Beauty and the Beast line in which they hired a young girl with Vitiligo who had been mentored by Winnie. This ad was very special for the Vitiligo community at large with many people taking pictures with the ad showing their own Vitiligo and then posting to social media.

During this time Vitiligo was buzzing in Social Media. The Magazine Allure released an interview of a woman with Vitiligo talking about her own journey. Several online articles were written about people with Vitiligo who were inspiring others through their fearless selfconfidence. In particular animals with Vitiligo were put in the spotlight, such as White Eyed Rowdy a black lab which resided in Portland Oregon. His family set him up social media profiles to help spread awareness about Vitiligo which he developed later in his life. Through this profile they raised charity money and connected him to the greater population of children with Vitiligo. Several stories involving him were seen in the media. Through the years Vitiligo has gone from a lesser known condition to one that people are much more likely to know about based on the current trend of awareness. This is helping people with Vitiligo to feel less alone as they get to see themselves represented more in the media.

Vitiligo is a skin condition which results in the progressive loss of pigmentation (color) of the skin. Vitiligo can often go unreported as it can be concealed, but it is said to affect approximately $.4-2 \%$ of the world population (Silverberg 2015). It affects all races and can occur at any time in a person's life. Prior to the emergence of social media, it was hard for people with Vitiligo to find others like themselves. They may have been separated geographically or chose to keep their condition hidden from the rest of the world. Previous research on Vitiligo has looked into the stigmatization involved with the condition and effects on self-esteem, examples including changes in behavior, cognitive ideas, and racial identity (Thompson, Kent, and Smith, 2002; Porter and Beuf, 1991).

Stigmatization is particularly salient when the attribute is visual as with Vitiligo. Stigma results 
from an attribute that is deeply socially discrediting causing an individual to not fit into normal society (Goffman 1963). This deviation from the norm allows the general society to classify a person in an undesirable way upon meeting them. Stigmatization makes it hard to gain full social acceptance. Stigmatized people are constantly aware of what makes them different leading to attempts to adjust their social identities. Vitiligo is often covered for one to pass as "normal." Covering inadvertently perpetuates the stigma. The condition will not become "normalized" if society never sees people with Vitiligo.

When it comes to people who have been stigmatized new media may allow for another resource for support. New media has received increasing research attention, as scholars try to understand the effect that it has on society. Studies are currently examining whether it has a net positive or negative effect on self-esteem. New media in relation to self-esteem has been shown to allow people to always feel connected to their "portable communities," receive support via "likes," and allow marginalized communities to come together (Chayko, 2008; Burrow and Rainone, 2016). This paper looks at how people with Vitiligo which are a marginalized group use social media to form community. Instagram is an example of a visual social media app which is being used by people with a visual skin condition.

Instagram is a social media app that currently has 800 million users worldwide who can post pictures and videos along with hashtags to make them accessible to the greater Instagram community (Instagram 2017). Hashtags are a word or phrase preceded by \# (pound sign) allowing users on social media to classify their post as a specific topic (Oxford Dictionary 2017). Approximately one-third (32\%) of all online adults report using Instagram (Greenwood, Perrin and Duggan 2016). When comparing the five most popular social media sites, Instagram ranked 2nd, far below Facebook (79\%), but slightly above Pinterest (31\%), LinkedIn (29\%), and Twitter (24\%) in use among online adults (Greenwood et al. 2016). Use of Instagram is highest among young adults 18-29 (59\%) with more female users overall (Greenwood et al. 2016). Use of Instagram also varies by race. More users identify as black (38\%) and hispanic (34\%) in comparison to white (21\%) (Krogstad 2015). The visual nature and use of hashtags makes this site an intriguing medium for this particular study which will look at the experiences of people who have a condition which is visual.

New media and Vitiligo were brought together in this study in ways that they haven't been previously. Interviews analyzed themes relating to the emotional experiences of people with Vitiligo who use Instagram. Taking into consideration the previous research mentioned on the topics of new media and Vitiligo. Looking particularly at the main themes of Vitiligo: behavioral changes, cognitive ideas, racial identity, and stigmatization in relation to themes found in the medium of new media.

The current study expanded on previous research by analyzing emotional experiences and compiling themes in relation to use of Instagram by people with Vitiligo. This was all in an attempt to better answer the question, What is the role of social media in the emotional lives of people with Vitiligo? This study found that support through Instagram did not translate into intense, close relationships but was constituted by what sociologists might refer to as weak ties. Granovetter's theory of weak ties refers to ties in which there is some mutual acknowledgement that the relationship is more than just being acquainted, but lacks the depth of a strong tie where there is a greater mutual investment (1973). Even though the ties are weak they provided 
immense support for participants. Through Instagram, people with Vitiligo are able to see others like themselves out there living their everyday lives. Participants took solace in the presence of others, gaining support through follows, likes, and comments which lead to an overall positive experience. Prior to social media, most people with Vitiligo felt alienated as there was no reflection of this part of them in daily life, nor a way to search out others. Hashtags have made searchability extremely easy to find others and participate. The community works in a way that allows people to find each other, see positive emotions and create a community based on weak ties.

\section{Literature Review}

Through previous literature on Vitiligo, stigma, and the effects of new media the story of how the Instagram-Vitiligo community works begins to form. People with Vitiligo feel stigmatized based on their condition. They are now able to find support through new media that previously was not easily accessible. This allows users to feel connected as they have a place where they can see themselves reflected by others in everyday life.

\section{$\underline{\text { Vitiligo }}$}

People with Vitiligo face various emotions after diagnosis. Coping with a condition which makes a person visually different can lead to adjustments in the way a person thinks, acts, and feels. These emotions result in common themes such as changes in behavior, cognitive ideas, and issues relating to racial identity.

The thought of being different seems to always be preoccupying people with Vitiligo. Even when it seemed participants in one study of experiences began to accept their Vitiligo, there was still an underlying awareness of the fact they were different (Thompson et al. 2002). These concepts are important as even when a person comes to acceptance of their Vitiligo, the stigma of being different may still always be in the back of their mind. In order to cope with the thoughts participants with Vitiligo used cognitive strategies. These cognitive strategies included minimizing thoughts of how noticeable their condition may be to others, attributing stares as a problem with that person's character or due to human nature, and social comparisons of how the situation could be worse (Thompson et al. 2002).

Due to the very visual nature of the condition, people with Vitiligo change their behavior to ease the anxiety associated with interacting with others. People with Vitiligo experience what Goffman (1963) refers to as Phantom Normalcy, where the stigmatized person must tip-toe around their stigmatized identity within society. They must attempt to fit in, while being fully aware they are not what society deems "normal," and not speak openly or passionately about their condition. People with Vitiligo often participate in concealing behaviors such as makeup or clothing. Special clothing to cover could include a combination of hats, gloves, long stockings, and long sleeves even in warm weathered months of the summer (Halder, Pham, and Crawford 1996). Wearing clothing which is inappropriate for the weather, such as a long sleeve shirt in the summer, can also garner attention as it appears abnormal. It can leave a person with Vitiligo unsure how to properly cover their condition without worry of being found out. This fear of 
being stigmatized can lead to social withdrawal (Thompson et al. 2002). One study suggested that by relying on these avoidant behaviors people with Vitiligo may be inadvertently maintaining their fear of stigma by not approaching it head on (Kent 1999).

Race plays an important role in people with Vitiligo. Vitiligo tends to be more noticeable in people with dark skin tones which can lead to further struggles. One study found that there was not a difference in the degree of disturbance due to stigma associated with having Vitiligo based on if the participant was black or white (Porter and Beuf 1991). Even though the degree of disturbance was not different, one issue that distressed black participants but not white participants was the idea of becoming white, as it conflicted with their racial identity. This study shows that for some people there may be more than just feelings of being stigmatized, but that an integral part of their identity is being affected. People may feel as if their racial identity is being robbed as they turn a very pale shade of white. This leaves a person with Vitiligo often struggling to find other ways that they identify as their particular race than just their skin color.

\section{Theory}

Vitiligo can be understood sociologically through Erving Goffman's idea of stigma (1963) which relates to an attribute that is deeply socially discrediting causing an individual to not fit into "normal" society. This deviation from the norm leaves other people in society to pre-judge a person upon meeting and classify them in an undesirable way. People who are stigmatized have trouble gaining full social acceptance. They are constantly aware of what makes them different leading to attempts to adjust their social identities. This constant impression management coupled with Phantom Normalcy leads to issues for the stigmatized person.

When it comes to describing the issues that people with Vitiligo often face stigma is salient. People with Vitiligo are constantly dealing with managing themselves in hopes of passing as "normal" which leads to further anxiety. The visual nature of the condition means that it can be covered, but only to a certain extent. People with Vitiligo may engage in wearing makeup or covering up with clothing. Makeup may lead to anxiety about it rubbing off or looking unnatural. Wearing covering clothing such as a long-sleeved shirt will look odd if the weather is hot leading to further stigmatization. A fear of being found out persists for those who choose to try to blend in. Through new media stigmatized individuals are able to break away from the constricting effects of "normal" society to seek out others for possible support.

\section{$\underline{\text { New Media Effects }}$}

The internet has the ability to bring people together in ways never thought possible in the past, allowing for possible connection and support across geographical lines. There are three major claims in the literature when it comes to the relationship between self-esteem and new media. These three claims include: allowing people to always feel connected to their "portable communities," receiving support via "likes," and allowing marginalized communities to come together (Chayko, 2008; Burrow and Rainone, 2016).

The "cognitive face" of communities explains that all communities (not just ones that exist on the internet) must be maintained in our minds (Chayko 2008). Even when people are distant from 
each other physically, as in online communities, then they are still cognitively present allowing them to feel very connected as they would in real life. People can gain satisfaction from the fact that their "portable communities" are easily accessed at any time of any day (Chayko 2008). For some this can create a greater connection as they always feel in contact with others. Through these online outlets people are able to construct an identity that represents themselves in whatever way they would like to be seen. The internet allows for a new way for socialization which can impact our identities as we battle with who we want to be seen as to others.

In relation to social support, the amount of "likes" received also seems to have an effect on the self-esteem of the social media user. When using Facebook as a social media platform the more "likes" received the higher the self-esteem of the individual (Burrow and Rainone 2016). Although, they did find if the person had a stronger sense of "purpose" in their life then they were less likely to have their self-esteem changed by the amount of "likes" received. A sense of purpose is defined in the article as, "self-organizing life aim that organizes and stimulates goals, manages behaviors, and provides a sense of meaning" (Burrow and Rainone 2016:1).

The internet has made it so social connections can be made without the bounding effects of geography. Prior to the emergence of the internet, if people with a shared interest or issue wanted to find each other then the process would be much harder due to the necessity to reveal their identity and the fact that they would be restricted to who was in their immediate area. In the age of the internet, a person can choose to follow others who are experiencing similar issues as happened in a study of cancer-based networks on Twitter (Murthy 2013). In this study individuals used the network to create a community not only to gain support, but also to ask questions relating to their own medical treatment. The internet creates a safe place where the stigmatized individual can garner the support of many to find information in relation to their own issue.

\section{Methods}

This sample consisted of 21 users of Instagram over the age of 18 that had profiles with pictures of Vitiligo visible. 17 participants identified as female and 4 as male. Ages varied with all participants ages ranging from 18 to 44 (7 participants in 18-24 range, 11 participants in 25-34 range, and 3 participants in 35-44 range). Participants varied in race/ethnicity in which they selfidentified. 5 participants identified as Black or African American, 6 as Hispanic, 5 as Caucasian or White, 1 as Indian/Asian, 1 as Southeast Asian/White, 2 as Native American, and 1 as Black/Puerto Rican. All participants were high school graduates and 11 identified as having pursued higher education. 4 participants had some college - no degree, 2 had an associate's, 4 had a bachelor's, and 1 had a graduate degree. Participants varied in amount of followers at point of contact on their Instagram profiles ranging from 141 followers at the lowest to 4,437 followers at highest. 1 participant had Instagram since the launch year of 2010, 7 started in 2012, 4 in 2013, 3 in 2014, 2 in 2015, and the remaining 4 participants had started last year in 2016. All participants in this study developed Vitiligo before age 28. Two-thirds of the participants (14) developed Vitiligo at age 13 or before. The rest (7) developed Vitiligo between the ages of 19 and 28.

A snowball sampling method was employed. To locate the sample for interviews, I searched the hashtag \#Vitiligo on Instagram. I direct messaged profiles that hashtagged \#Vitiligo and also had 
posted a picture of visible Vitiligo until I had enough people agree to participate in order to fulfill my sample. I interviewed participants via video calling software or instant messaging software (e.g. Facetime, Google Hangouts). I direct messaged participants through Instagram with a general explanation to see if they were interested in participating. Then if they said they were interested, I would exchange messaging info and set up a time with them. During the interviews, I went over the consent form then proceeded with my questions after receiving consent. I recorded the video calling interviews after asking for the participants consent to do so. A total of 7 participants were able to be video interviewed and the other 14 were done through instant messaging. All interviews were conducted during 2017. The interviews were semistructured interviews consisting loosely of the questions below and changing depending on the conversation.

1. When did you first notice your Vitiligo?

2. When did you start using the hashtag Vitiligo and why?

3. Do you have friends that don't use the hashtag Vitiligo and why?

4. How did you hear about the hashtag?

5. Can you tell me about your history with Instagram?

6. Walk Participant through the last 3 Instagram posts with Vitiligo visible asking:

- Why did you decide to post this?

- What were some of the reactions?

- How did that make you feel?

7. Do you think Instagram has affected how you feel about Vitiligo?

8. Has someone ever reacted in a way that made you feel bad about yourself tell me about that?

9. Has someone ever reacted in a way that made you feel good about yourself tell me about that?

\section{Findings}

When beginning this research, I expected to find that participants were making strong connections with other users. I actually found out that that the support in this community was provided through weak ties. Even though these ties were what sociologists refer to as weak, they provided tremendous support for participants. They felt good just knowing that there were others like themselves out there living their everyday lives. Prior to social media, most people with Vitiligo felt alienated as there was no reflection of this part of them in daily life, nor a way to search out others. Hashtags have made it extremely easy to find others and participate. Participants mainly take solace in the presence of others, rather than making strong connections. The community works in the way that allows them to find each other, see positive emotions and create a community based on weak ties. 


\section{Hashtags}

Hashtags have become so enmeshed in today's culture that it is now defined in the dictionary. Hashtags are a word or phrase preceded by \# (pound sign) allowing users on social media to classify their post as a specific topic (Oxford Dictionary 2017). This has been extremely useful in regards to Vitiligo and Instagram. The ease of access and searchability for others with Vitiligo has never been so uncomplicated. When asked about the hashtag \#Vitiligo participants were delighted to tell me about the first time that they searched the hashtag and realized there were so many people like themselves out there.

In regards to asking how she heard about \#Vitiligo Vivica said, "I think I hashtagged it myself and then searched it. Just to see. And I realized like a whole lot of people were using it." She went on to say, "I had seen people with Vitiligo before, like one or two, but I didn't even realize there were so many people out there and just a lot of pictures." When asked about hashtags participants echoed Vivica's response. Once participants figured out that hashtags were filters that could easily bring them to others with Vitiligo they began to actively participate in using the hashtag and searching it. By participating in the Vitiligo community with the hashtag they then gain support through likes and followers. Participants also used variations of hashtags for selfexpression. Some users had some variation of their screen name as a hashtag preceded by Vitiligo. There was a theme of adding Vitiligo to words such as \#VitiligoBeauty, \#VitiligoGirl, or \#VitiligoModel. One participant said her favorite tag variation at the moment added the word kween (queen) to Vitiligo. She explained her use of kween was to make reference to the pop culture use of the word kween (queen) on the show Broad City.

Participants were also concerned about using the correct hashtags to reach the broadest audience possible. Christian mentioned this concern when I asked about the thoughts and feelings that go through his mind when posting a picture with Vitiligo visible to Instagram. "I'm just thinking, like I'm hoping, I'm using all the right hashtags. Like I hope that people can see this." He went on to explain that when growing up there was overwhelming stigma and kids would pick on him for his Vitiligo. He feels that after Winnie Harlow went on Top Model it really changed the view for people with Vitiligo. He then went on to speak of pride in posting a picture and how he hopes it reaches people. "It makes me so proud like when I put it on there. I just hope that people see it, you know what I mean?" Hashtags were often used in this way to help fight stigma and spread awareness. Tags such as \#VitiligoProud, \#NoCureDontCare, and \#SoRareTheyJustStare had a motivational theme that there was nothing wrong with being a person with Vitiligo. Tags such as \#VitiligoAwareness and \#ItsCalledVitiligo are aimed at spreading the word about the condition. Uniting tags were also used such as \#VitiligoNation and \#InOurSkinWeWin to make people feel less alone and that they are part of a larger community.

Hashtags have been an integral part of the Vitiligo community forming on Instagram. Not only have they brought people together as a search tool, they have gone further in allowing users selfexpression and the ability to spread positivity to fight stigma associated with their condition.

\section{Positivity}


When it came to Instagram, I was very interested to see how others perceived the experience of Instagram. Did they find it to be positive or negative? What types of communities were forming in relation to Vitiligo? Were people "trolling" as seen on other social media sites. "Trolling" in the internet sense is when offensive posts are made purposefully by users in order to elicit a response or harass others online. What I found was quite interesting, there was an overall expression of Instagram being a positive place. Participants often told me of positive experiences of being involved with Instagram before I could even ask them specifically about it. Positivity was talked about on Instagram in instances directly comparing to others social media sites such as Facebook which were perceived as more negative. Part of this lack of negativity may have to do with the format of Instagram. On Facebook when a user posts, it is seen by everyone on their friends list who follows them. When a person comments it alerts everyone else who has commented previously. This makes for a more back and forth conversation between all parties as it is constantly updating everyone on where the conversation is at currently. This is great, except when people get involved in the conversation who are looking to "troll." Then this constant updating means that more negativity can easily spread as no extra steps are needed to alert more people which a response may be elicited from. Instagram is different in that it will not alert everyone who has commented when another user comments. The only way to keep a back and forth conversation going in a comment section is to specifically tag someone in the conversation by using the "@" symbol and then the user's screen name with every comment. While it is possible to "troll" on Instagram, the process is a lot more cumbersome than on other social media sites and may not get as much of a response which may lead to it being a less attractive app to "troll." Instagram recently launched a campaign called Instagram-Together to break down stigma in relation to mental health promoting that users find their support community on Instagram. On their website Instagram-Together.com they give ways to keep your community safe through tools that allow for moderating what others can see and comment. These safety tools along with the format of the app are part of what is contributing to the positive vibe that the current participants have mentioned allowing for a safe space on the internet.

When I asked if participants if they had negative experiences on Instagram, there was usually a moment to think about it and then they explained no, it was positive. The participant would then generally tell me a story from everyday life or elsewhere that they had been stigmatized based on their condition. Pica told me about a lot of positive feedback that he had experienced on Instagram and when I asked if anyone had ever reacted in way that made him feel bad on Instagram he responded with, "On Instagram? No. I have had negative feedback in real life though. And even on my Facebook page, but here I haven't really had no negativity thrown at me so far. People seem to be more open minded on IG."

Of my 21 participants, only 1 participant said she had what was perceived to be a negative reaction on Instagram. The negative reaction was from a user asking why she was putting white makeup on her face. These types of comments are disconcerting but luckily not the norm with participants I interviewed. Another participant described interactions that aren't so much negative but more what she described as well intentioned people misunderstanding. For example she may caption a picture of herself with Vitiligo as something such as "My Vitiligo is poppin' today!" to which users will react with something along the lines of, "No, you're still beautiful even with Vitiligo." She explained these comments though well meaning are defeating when she 
is trying to celebrate her Vitiligo, but society can only see it as a flaw. This is comparable to the type of backhanded compliments our society deems on other stigmatized groups. An example of this would be the commonly used, "You're cute for a ..." this blank could be inserted with various characteristics that society considers undesirable. Society often sees the two things as opposing to where the beauty in someone has to be seen despite the supposed undesirable characteristic. This type of language sends the idea that the person still doesn't live up to societal standards.

When looking through participants comments on pictures I saw overwhelming positivity. The only times that I did see negativity start to creep into Instagram was not on personal profiles but on advertisements which included people with Vitiligo. Winnie Harlow's ad on Converse was met with some negative comments. Users who follow Converse on Instagram and may not be familiar with Vitiligo were suddenly faced with it in their Instagram feed. Converse did not offer an explanation of what Vitiligo was in their caption or even hashtag Vitiligo, which it could be argued either way whether they should have or not. On one hand it is good to offer an explanation so the general public understands if they haven't seen Vitiligo before. On the other hand it seems if the company pointed it out then it may be offensive as it would not be necessary to point out things about a model in a usual situation. Although the majority of comments were positive, this lack of explanation and users suddenly seeing Vitiligo led to some comments which were negative such as, "creepy" or "WHAT IS HER SKIN? ." There was also evidence of some trolling which was moderated as users kept tagging a specific user explaining how it was not right for him to say things like that about Winnie Harlow, but his comments were no longer in the feed.

Positivity was seen in hashtags as mentioned earlier but was also strongly present in the captions of photos. Captions were used for inspirational quotes such as making it through a tough time. Participants also used captions for sharing a piece of their own journey with Vitiligo that has led to self acceptance. Some participants had Instagram accounts which were specifically aimed at Vitiligo where they only posted Vitiligo centric posts. These profiles had themes of acceptance and awareness. Other participants had profiles that were more enmeshed with their daily lives. They still posted about their Vitiligo but it was not the main topic they were focused on. Specific profiles are seen throughout Instagram in all different types of topics, an example would be an Instagram account only for pictures of fitness/working-out as opposed to a user having an account where they post pictures of other things in their lives along with fitness pictures. Profiles that are specified on one topic can target a specific audience possibly leading to greater followers/likes. Profiles which include photos of other daily happenings unrelated to Vitiligo can lose interest from followers if they don't care about other aspects of the user's life. Emerging out of this specificity are specific profiles which users with Vitiligo make and manage re-posting only pictures of other users they find via Instagram with Vitiligo. These profiles celebrate users with Vitiligo by adding a more direct link to see pictures of others with Vitiligo in the Instagram feed without just searching the hashtag. These types of profiles also add to the positive community aspect as a user having their picture displayed by one of these profiles is another layer which can feel like induction further into the community. Due to the specificity of these profiles they can garner many followers leading to a picture receiving more positive reinforcement through likes and comments. 


\section{$\underline{\text { Weak Ties }}$}

When setting out to perform this research, I hypothesized that I would find that participants were developing strong relationships where they were receiving deep support from others with Vitiligo. On the contrary, I found that participants were not developing these strong ties, but rather what is referred to as weak ties. Granovetter's theory of weak ties refers to ties in which there is some mutual acknowledgement that the relationship is more than just being acquainted, but lacks the depth of a strong tie where there is a greater mutual investment (1973). A form of these weak ties was found in the Vitiligo Instagram community. Participants still felt very supported by the Vitiligo community on Instagram, but it was through follows, likes, and comments. People on Instagram make connections by following others which can be reciprocated, but the connections generally do not blossom further than likes and comments through Instagram. The community works in the way that allows people with Vitiligo to find each other, see positive emotions and create a community based on these weak ties.

Amanda explained that establishing friendships on Instagram was harder than she expected, "I follow a few different IG accounts that feature many different people with vitiligo, and despite commenting on people's photos, I have yet to really talk to someone and form a friendship." Ellie responded similarly when asked if she felt she had made "friends" with people through Instagram, "Not really friends, but you meet people all over with the same life as you, so I'd say I meet a lot of people but do not become acquainted past Instagram. I haven't been so lucky yet. ;)." Participants appreciated knowing that there were a lot of other people out there like themselves living everyday lives. Even though Vitiligo affects up to $2 \%$ of the global population it would have been very hard to find anyone else with Vitiligo in the past (Silverberg 2015). People in daily life may choose to cover up their Vitiligo making it so that two people with Vitiligo could interact without ever knowing that the other has Vitiligo. Through Instagram users are able to see others like themselves in a few easy swipes. This feeling of not being alone was something that was echoed by all participants. Instagram is allowing for the representation that is not often seen in everyday life. Participants follow other users with Vitiligo and get to feel connected in their lived experience as they post of their own successes and struggles. They offer support through positive reinforcement of likes and comments. There is a friendly relationship but not usually one that moves past the usual likes and comments.

Amelia told me of her first Vitiligo themed post on Instagram. It was of her hands showing her Vitiligo. She explained she was having a really bad day and she just wanted to post a picture to remind herself of how beautiful her hands were, not by appearance but by what they could do. She told me of the various activities that she does that require dexterity such as knitting, rock climbing, writing, and playing musical instruments. She posted the picture of her hands along with a caption explaining how she thought her hands were beautiful because of this and hashtagging Vitiligo. This led to a positive outcome for her. Amelia recalled, "I started to get like a ton of Vitiligo related followers and I was like 'Oh My God,' like at that point, I had the whole like 'Oh Shit,' there are other people like me out there!' Amelia's excitement is a good example of the type of response I would get from participants when recalling finding others with Vitiligo through Instagram.

While it wasn't common for participants to make deep connections through Instagram, 2 out of 
the 21 participants did mention this happening with a few of their followers. One participant met her partner which she refers to as her soulmate through Instagram. Her partner which does not have Vitiligo but is an admirer of Vitiligo was searching the hashtag Vitiligo when he came across her profile. He was able to establish contact with her through Instagram where their relationship slowly built. He only lived a state away and they eventually met up. This relationship blossomed and he eventually moved to her state to live with her. They now have a young child and their relationship is still going strong. The other participant who had deeper connections was able to meet two twin young women with Vitiligo her age from a neighboring high school. They all participated in theater competitions and were busy scoping out the competition's Instagram when they noticed each others Vitiligo. After this they made contact and realized they had a lot in common in addition to Vitiligo. Since then they have maintained contact. The participant said she only sees them a few times a year but she definitely thinks of them as her best friends, but much more like sisters than her best friends.

\section{Conclusion}

The current study on the Vitiligo Instagram community brought up three main ideas in relation to stigmatized groups which were hashtags, positivity, and weak ties. The community is able to form because hashtags on the visual app make it extremely easy for people with Vitiligo to find one another. Before social media, it was very difficult to find another person with Vitiligo due to geographical constraints and societal stigma that often makes an individual feel like they have to conceal. In addition to searchability, participants were able to use these hashtags for selfexpression and motivation. Instagram was perceived as an overall positive community by participants. Part of this having to do with format of the application itself with its options for moderation. The other part being the way that the positive community is fostered through captions which display inspirational quotes, stories, and hashtags. Users give each other positive reinforcement through likes and comments on posts displaying Vitiligo. The support that is received through Instagram is done through weak ties. Users do not develop intense, close relationships but rather get support through just the presence of others. The community allows people with Vitiligo to not feel alone. Users feel a connection in a lived experience and get to see others like themselves living their lives. They themselves can also feel good when they post as they are contributing to the awareness and the community at large.

\section{Future Research}

During the course of this research Vitiligo became more well known it also started to be considered "trendy." Instagram has a prominent beauty culture where users post their beauty techniques and tutorials. As Vitiligo gained awareness more people began taking on Vitiligo as a beauty trend. This could be seen through makeup application or digitally altering photos to appear that the user has Vitiligo. One company on Instagram was even selling high-fashion gloves that one could wear to mimic Vitiligo as if it was a print like "cheetah." During the process of working on this research I also began interviewing participants in relation to how they feel about this Vitiligo beauty trend while also watching the comments unfold on Instagram each time a new person decides to "try-on" Vitiligo. I was seeing mixed views from the Vitiligo Instagram community. Some interviewed compared this trend to "blackface." The user simulating Vitiligo gets to just "try-on" Vitiligo without understanding the underlying oppression 
and stigmatization that comes along with living with the condition. This leads to some backlash from people in the Vitiligo community towards the person who is following the beauty trend. Other participants are not offended, but just happy to have further awareness as the community has gone so long stigmatized and unrecognized. The movie Deadpool 2 will be released in 2018 which features a character named Domino which will have a Vitiligo patch around one of her eyes. The person playing the character does not have Vitiligo so makeup is being applied for the effect. The addition of a Vitiligo character in a wide release superhero movie will mean that there will be even more awareness brought to Vitiligo, but it also means people without Vitiligo will potentially cosplay the character by applying makeup as if they have Vitiligo. Further research over time will tell how Vitiligo and faux application will be perceived by not only society, but people with Vitiligo themselves.

\section{Limitations}

These results cannot be generalized to the greater population due to the specific nature of the study. The only form of social media being examined is Instagram. It can not be determined if a person would use Instagram in the same way that they may use another social media website. Instagram is a completely visual site where users can only posts photos or videos along with captions which can have hashtags to reach a broader community of users. Users can elect to follow other users without expecting a follow back as opposed to traditional sites such as Facebook where people become "friends." This may allow for a more informal way of communicating with other users who are not a person's real life friends, acquaintances, or friends of friends. It is also important to point out the app is constantly evolving and adding new features. At the time of interviewing participants for this study, Instagram's main features included posting pictures/videos, liking, commenting, and direct messaging. This study was done with a small sample from a subset of the population that use Instagram which have the particular skin condition Vitiligo. A nonprobability sampling method was used, which may mean something about the type of subject willing to participate in this study (e.g. only people who have good experiences on Instagram). This sample mostly consisted of female participants. More sampling would have to be done to obtain broader views from people of all genders. The views expressed by the participants may not be reflective of not only the greater population of people with Vitiligo, but even people with Vitiligo who choose to use Instagram. 


\section{REFERENCES}

Burrow, Anthony L. and Nicolette Rainone. 2016. "How Many Likes Did I Get?: Purpose Moderates Links between Positive Social Media Feedback and Self-Esteem.” Journal of Experimental Social Psychology.

Chayko, Mary. 2008. Portable Communities: The Social Dynamics of Online and Mobile Connectedness. State University of New York Press.

Goffman, Erving. 1963. Stigma; Notes on the Management of Spoiled Identity. Englewood Cliffs, NJ: Prentice-Hall.

Granovetter, Mark. 1973. "Strength of Weak Ties.” Encyclopedia of Social Theory 1360-80.

Greenwood, Shannon, Andrew Perrin, and Maeve Duggan. 2016. "Social Media Update 2016." Pew Research Center: Internet, Science \& Tech. Retrieved December 12, 2016 (http://www.pewinternet.org/2016/11/11/social-media-update-2016/).

Halder, Robert M., Hoang N. Pham, and Lynda M. Crawford. 1996. "Vitiligo: a Life-Altering Skin Disease." Loss, grief \& care 7(3/4):153-60.

Instagram. 2017. "Strengthening Our Commitment to Safety and Kindness for 800 Million." Instagram Business. Retrieved January 31, 2018 (https://business.instagram.com/blog/safety-and-kindness-for-800-million/).

Kent, Gerry. 1999. “Correlates of Perceived Stigma in Vitiligo.” Psychology \& Health 14(2):241-51.

Krogstad, Jens Manuel. 2015. "Social Media Preferences Vary by Race and Ethnicity.” Pew Research Center. Retrieved December 12, 2016 (http://www.pewresearch.org/fact$\operatorname{tank} / 2015 / 02 / 03 /$ social-media-preferences-vary-by-race-and-ethnicity/).

Porter, Judith R. and Ann Hill Beuf. 1991. "Racial Variation in Reaction to Physical Stigma: A Study of Degree of Disturbance by Vitiligo Among Black and White Patients." Journal of Health and Social Behavior 32(2):192-204. Retrieved November 2, 2016 (http://www.jstor.org/stable/2137152).

Murthy, Dhiraj, Alexander Gross, and Daniel Oliveira. 2013. "Understanding Cancer-Based Networks in Twitter Using Social Network Analysis." in Twitter: social communication in the Twitter age. Cambridge: Polity.

Oxford Dictionary. 2017. “Oxford Dictionary Hashtag." Retrieved May 22, 2017 (https://en.oxforddictionaries.com/definition/hashtag)

Silverberg, Nanette B. 2015. “The Epidemiology of Vitiligo.” Current Dermatology Reports 4(1):36-43. 
Thompson, Andrew R., Gerry Kent, and Jonathan A. Smith. 2002. "Living with Vitiligo: Dealing with Difference." British Journal of Health Psychology 7(2):213-25.

\section{Acknowledgements and Author Information:}

Elizabeth Witcher produced this work as an undergraduate honors thesis for the University of Oregon sociology department during the 2016-2017 academic year advised by Dr. CJ Pascoe. This work was adapted further as part of the McNair Scholars Program advised by Dr. CJ Pascoe (Thesis Advisor) and Dr. Gail Unruh (Program Director) with the final submission of the thesis made to the McNair Scholars Program in January 2018. All names above are pseudonyms as is standard in sociological research to protect the identities of those interviewed. This work would not have been possible without the willingness of those in the Vitiligo community to communicate their stories. Elizabeth is insider in the community studied and has had Vitiligo since she was 3 years old. The author is forever grateful to those who participated as well as those who mentored her along the way. Elizabeth began at the University of California, Davis as doctoral student in sociology in September 2018. 